Research Article

\title{
Chiral All-Dielectric Metasurface Based on Elliptic Resonators with Circular Dichroism Behavior
}

\author{
Ali Yahyaoui $\mathbb{D}^{1,2}$ and Hatem Rmili $\mathbb{D}^{1}$ \\ ${ }^{1}$ Electrical and Computer Engineering Department, King Abdulaziz University, P.O. Box 80204, Jeddah 21589, Saudi Arabia \\ ${ }^{2}$ Electrical and Computer Engineering Department, University of Jeddah, P.O. Box 80327, Jeddah 21589, Saudi Arabia
}

Correspondence should be addressed to Hatem Rmili; hatem.rmili@yahoo.fr

Received 26 November 2017; Revised 5 February 2018; Accepted 8 February 2018; Published 19 April 2018

Academic Editor: Giuseppe Castaldi

Copyright (c) 2018 Ali Yahyaoui and Hatem Rmili. This is an open access article distributed under the Creative Commons Attribution License, which permits unrestricted use, distribution, and reproduction in any medium, provided the original work is properly cited.

\begin{abstract}
We present the design of a new microwave all-dielectric chiral metasurface with circular dichroism behavior based on elliptic dielectric resonators (EDRs). During the design procedure, we have first optimized numerically the effects of the main design parameters such as the resonator's size and orientation, as well as the size of the slot within them, on the metasurface transmission over the frequency band $10-20 \mathrm{GHz}$. Measurements on the final metasurface prototype have shown a large circular dichroism $(\Delta=0.61$ and $\Delta=0.5)$ over two bands $(17.55-17.61 \mathrm{GHz}$ and $17.91-18.05 \mathrm{GHz})$ with ellipticities close to $45^{\circ}$ which means that the developed device can be used as a circular polarization filter and circular polarizer at microwave frequencies.
\end{abstract}

\section{Introduction}

Metamaterials are artificially media characterized by exotic physical properties which do not exist in natural materials [1-4]. These structures are usually obtained by arranging a set of subwavelength-scattering elements in periodic structures to ensure the coupling between electric and magnetic components of the incident electromagnetic fields. This special arrangement offers to metamaterials original electromagnetic properties that can be tailored to a given application which is very useful for the design of innovative microwave devices such as cloaks, lens, absorbers, and polarizers.

However, to overcome the main disadvantages of metamaterials such as high losses, bulkiness, and difficulty of fabrication, the scattering elements may be arranged in two-dimensional periodic structures, which are metasurfaces $[5,6]$. These $2 \mathrm{D}$ version of metamaterials have permitted to control the amplitude, phase, and polarization of electromagnetic waves, giving many other potential applications such as controllable surfaces, miniaturized cavity resonators, novel wave-guiding structures, angular-independent surfaces, absorbers, biomedical devices, fast switches, and fluid-tunable frequency-agile materials [7].

In addition, to further improve the performances of metasurfaces and avoid their ohmic losses, which may limit their use at higher frequencies, all-dielectric metasurfaces with low losses, high overall efficiency, and various functionalities [8-16] were developed for several microwave $\mathrm{THz}$ and optical applications. In all-dielectric metasurfaces, the main used scattering elements are dielectric resonators such as spheres, cubes, cylindrical/elliptical disks, and rods [7]. By using high dielectric constant dielectric resonators, we can excite both electric and magnetic resonant modes and reduce the metasurface size.

On the other hand, chiral metamaterials $[17,18]$ have attracted much attention due to their interesting properties such as optical activity [19,20], circular dichroism [21], and negative refractive index [20]. In these artificial media, the optical and circular dichroism properties are many orders of magnitude higher than those in natural chiral materials, whereas negative refractive index property can be achieved without combination of negative permeability and negative 
permittivity. In addition to these interesting properties, use of chiral metasurfaces instead of chiral metamaterials offers other original characteristics such as the asymmetric transmission of electromagnetic waves through the two opposite sides [22-24].

Within this context, we propose the design of a new alldielectric chiral metasurface with circular dichroism properties at microwave frequencies, based on elliptic dielectric resonators. Recently, we have used these resonators to design quarter-wave plate (QWP) and half-wave plate (HWP) metasurfaces operating at microwave frequencies $[11,15,16]$.

In this paper, we have studied numerically and experientially the electromagnetic response of an all-dielectric chiral metasurface over the band $10-20 \mathrm{GHz}$. The fabricated metasurface prototype showed large circular dichroism $(\Delta=0.61$ and $\Delta=0.5)$ over two bands centered on 17.56 and $18 \mathrm{GHz}$, respectively, with ellipticities close to $45^{\circ}$, permitting it to be proposed as circular polarization filter and circular polarizer.

\section{Theoretical Formulation of the Circular Dichroism}

The circular dichroism (CD) is one main property characterizing chiral metasurfaces. This effect is due to the transmission difference, in terms of absorption and polarization state, of the metasurface when excited by incident righthand circularly polarized (RCP) and left-hand circularly polarized (LCP) waves, respectively. The absorption $(\Delta)$ and polarization state $(\eta)$ parameters are given by the following equations [25]:

$$
\begin{aligned}
& \Delta=\left|T_{++}\right|^{2}-\left|T_{--}\right|^{2}, \\
& \eta=\frac{1}{2} \arcsin \left[\frac{\left|T_{++}\right|^{2}-\left|T_{--}\right|^{2}}{\left|T_{++}\right|^{2}+\left|T_{--}\right|^{2}}\right],
\end{aligned}
$$

where $T_{++}$and $T_{--}$are the transmitted coefficients of RCP and LCP waves, respectively.

The coefficients $T_{++}$and $T_{--}$are related to the linear transmission coefficients $\mathrm{T}_{x x}, \mathrm{~T}_{x y}, \mathrm{~T}_{y x}$, and $\mathrm{T}_{y y}$ (the first and second subscripts denote transmitted and incident polarizations, resp.) through the following equation [25]:

$$
\begin{aligned}
& T_{++}=\frac{1}{2}\left[\left(T_{x x}+T_{y y}\right)+i\left(T_{x y}-T_{y x}\right)\right], \\
& T_{--}=\frac{1}{2}\left[\left(T_{x x}+T_{y y}\right)-i\left(T_{x y}-T_{y x}\right)\right],
\end{aligned}
$$

where $T_{x x}=E_{x}^{t} / E_{x}^{i}, \quad T_{x y}=E_{x}^{t} / E_{y}^{i}, \quad T_{y x}=E_{y}^{t} / E_{x}^{i}$, and $T_{y y}=$ $E_{y}^{t} / E_{y}^{i}$. For example, $E_{x}^{i}$ is the $x$-polarized incident electric field and $E_{y}^{t}$ is the $y$-polarized transmitted electric field.

\section{Metasurface Design Procedure}

The all-dielectric chiral metasurface of thickness $5.12 \mathrm{~mm}$ is composed of two layers of EDRs of thickness $h=2.56 \mathrm{~mm}$ made of Rogers RO3210 (relative permittivity 10.2, loss

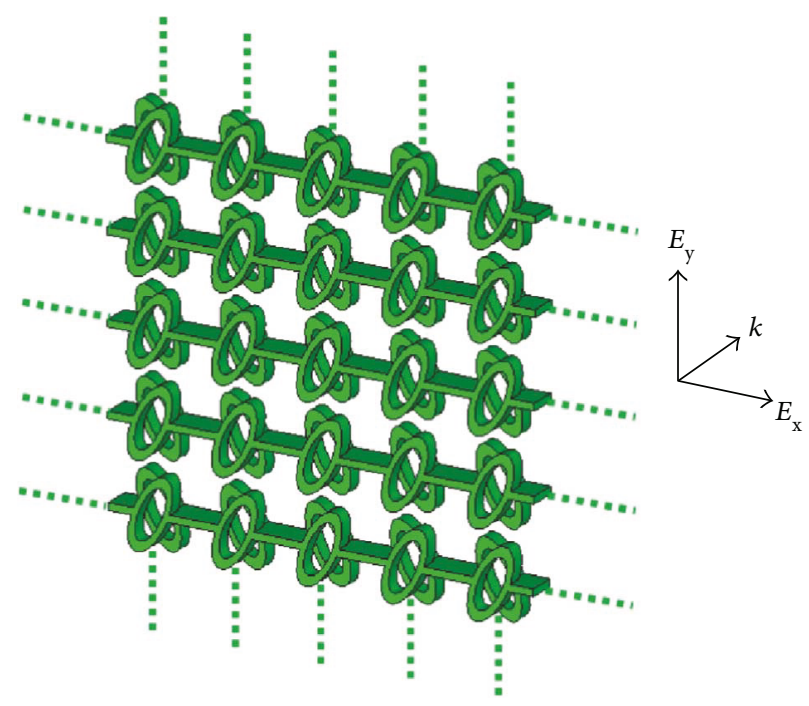

Figure 1: Perspective view of the all-dielectric metasurfaces with connected dielectric resonators along the $x$-direction.

tangent 0.0027 ) as shown in Figure 1. Each layer is composed with an infinite array of connected EDRs.

The dielectric ring resonator composing the unit cell was obtained by subtracting an elliptic volume (slot) of minor radius $a_{\mathrm{s}}$, major radius $b_{\mathrm{s}}$, and ellipticity $\tau_{\mathrm{s}}=b_{\mathrm{s}} / a_{\mathrm{s}}$ from elliptic dielectric resonators of minor radius $a$, major radius $b$, and ellipticity $\tau=b / a$. Both the elliptic resonator and slot are concentric. The resonators composing the upper and lower layers are rotated around their $z$-axis with angles $+\phi$ and $-\phi$, respectively, as shown in Figure 2, and connected along the $x$-direction with thin strips of length $L_{c}=\left(L_{x} / 2\right)-a$ and width $W=1.25 \mathrm{~mm}$.

The unit cell (Figure 2) is modelled by a box of dimensions $L_{x} \times L_{y} \times L_{z}\left(L_{x}=14.9 \mathrm{~mm}, L_{y}=14.9 \mathrm{~mm}\right.$, and $L_{z}=160 \mathrm{~mm}$ ), where the longitudinal dimension $L_{z}$ along the propagation direction $z$ is greater than the wavelength $\left(L_{z}=16 \lambda\right)$, and the transverse dimensions $L_{x}$ and $L_{y}$ along $x$ - and $y$-directions, respectively, are lower than the wavelength $\left(L_{x}<\lambda\right.$ and $\left.L_{y}<\lambda\right)$, with $\lambda=15 \mathrm{~mm}$ being the freespace wavelength associated to the upper frequency of the band 10-20 GHz.

We have used the electromagnetic simulator CST Microwave Studio to perform numerical simulation. The structure was excited under normal incidence with periodic boundary conditions along the $x$ - and $y$-directions.

We have studied the effect of the main design parameters such as the resonator ellipticity $\tau$, the slot ellipticity $\tau_{\mathrm{s}}$, and the orientation of the resonators described by the azimuth angle $\phi$ on the metasurface properties (see Figure 3 ). These parameters were optimized to improve the metasurface circular dichroism by analyzing the variation of the absorption $\Delta$ and the polarization state $\eta$ variation over the frequency band $10-20 \mathrm{GHz}$. The main data related to this study are regrouped in Table 1.

First, the effect of the resonator (without slot) ellipticity on the chiral metasurface transmission (see Figures 3(a) and $3(\mathrm{~b})$ ) was studied by fixing the resonator minor radius $a$ to $3 \mathrm{~mm}(\lambda / 5)$ and by varying the ellipticity $\tau$ (variation 


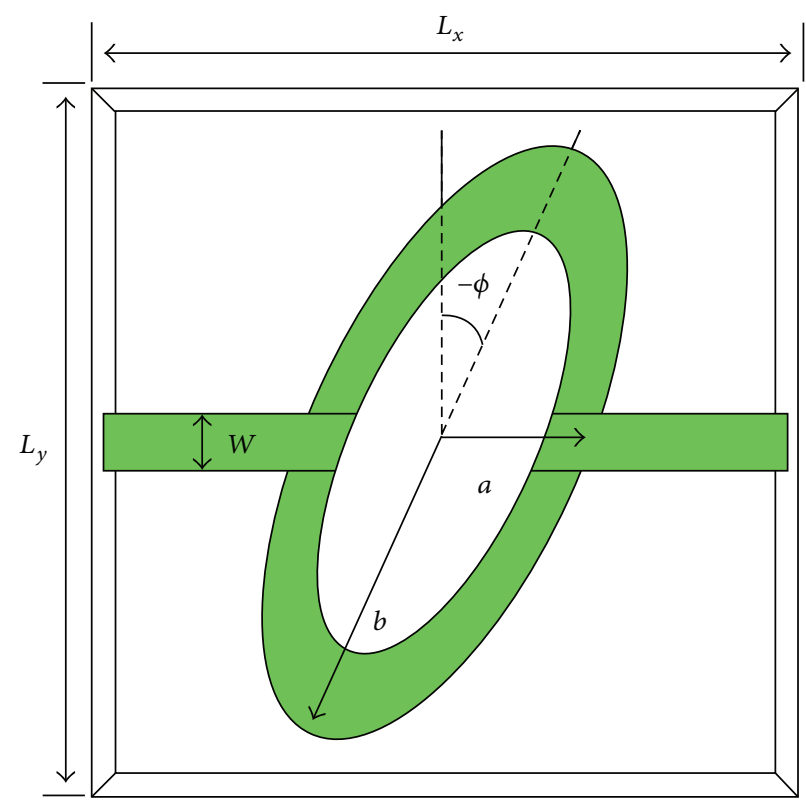

(a)

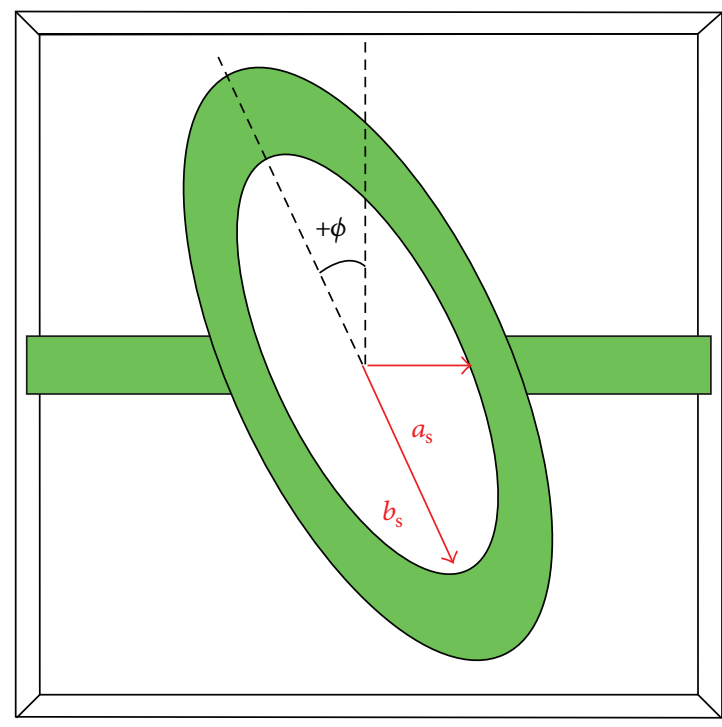

(b)

FIgURE 2: CST model for the unit cell: (a) upper layer; (b) lower layer.

of the major radius $b$ ) without affecting the cell size. The azimuth angle $\phi$ defining the resonator orientation was fixed to $25^{\circ}$. In Figures $3(\mathrm{a})$ and $3(\mathrm{~b})$, we have illustrated the variation of the absorption $\Delta$ and ellipticity angle $\eta$, over the frequency band $10-20 \mathrm{GHz}$, for different values of the ellipticity $\tau(\tau=1.33,1.66,2$, and 2.33), respectively.

Next, we tried to ameliorate the metasurface performances (absorption, bandwidth, and polarization of transmitted waves) by taking the previous optimized configuration $\left(\tau=2.33, \phi=25^{\circ}\right)$ and by varying the resonators' orientation; the resonators were rotated around their $z$-axis with an angle $\phi\left(5^{\circ}<\phi<45^{\circ}\right)$. Figures 3(c) and 3(d) show the variation of both the absorption $\Delta$ and ellipticity angle $\eta$ for the rotation angles $\phi=15^{\circ}, 25^{\circ}, 35^{\circ}$, and $45^{\circ}$.

Finally, to further improve the metasurface properties in terms of absorption, bandwidth, and ellipticity, we have added elliptic slots of ellipticity $\tau_{\mathrm{s}}$ to the resonators $\left(\tau=2.33\right.$ and $\left.\phi=25^{\circ}\right)$. Figures $3(\mathrm{e})$ and $3(\mathrm{f})$ illustrate the parametric study realized for the slot ellipticities $\tau_{\mathrm{s}}=1.5,2$, 2.5 , and 3 .

As it can be noticed from Table 1, from the four studied resonator ellipticities $(\tau=1.33,1.66,2$, and 2.33$)$, only the values $\tau=2$ and 2.33 showed high absorption levels (more than 50\%). The best configuration (with $\phi=25^{\circ}$ ) corresponds to $\tau=2.33$ with an absorption peak of 0.9 at $12.62 \mathrm{GHz}$ and ellipticity $\eta=38.13^{\circ}$.

On the other hand, the rotation of the elliptic resonators without slots may not improve their performances. For the circular dichroism, the best configuration remains at $\phi=25^{\circ}$ where absorption is high over the band $12.18-13.14 \mathrm{GHz}$ with a maximum of 0.9 at $12.62 \mathrm{GHz}$. Similarly, the ellipticity $\left(\eta=38.13^{\circ}\right)$ also was not improved significantly by varying the rotation angle $\phi$.

However, the addition of the slots has shifted all the resonating frequencies and improved the metasurface performances. For example, with a slot ellipticity $\tau_{\mathrm{s}}=2$, we obtain the best metasurface operating over the frequency band $16.82-18.76 \mathrm{GHz}$ (10.9\%), with maximum values of absorption $(\Delta=0.91)$ and ellipticity $\left(-44.6^{\circ}\right)$ at $17.24 \mathrm{GHz}$. Nevertheless, for the slot ellipticity $\tau_{\mathrm{s}}=2.5$, we obtain stronger circular dichroism $(\Delta=0.93$ at $17.91 \mathrm{GHz})$ but lower values of bandwidth $(7.5 \%)$ and ellipticity $\left(\eta=36.96^{\circ}\right)$. We can also notice a large ellipticity $\left(\eta=-44.4^{\circ}\right)$ for the metasurface designed with $\tau_{\mathrm{s}}=1.5$, but again, the other performances are lower $(\Delta=0.77$ with bandwidth equal to $9.1 \%)$.

From the previous results, we can conclude that the optimized metasurface designed with resonator ellipticity $\tau=2.33$, and orientation $\phi=25^{\circ}$, and with slots of ellipticity $\tau_{\mathrm{s}}=2$ showed high circular dichroism over the wide frequency range $16.82-18.76 \mathrm{GHz}(10.9 \%)$ with a peak of $\Delta=0.91$ at $17.24 \mathrm{GHz}$ and a large ellipticity close to $-45^{\circ}$ $\left(\eta=-44.6^{\circ}\right)$, which offers to the proposed microwave device the possibility to transmit circularly polarized waves and thus the capability to work as a circular polarization filter and/or circular polarizer.

\section{Experimental Results and Discussion}

The fabricated all-dielectric metasurface of length $74.5 \mathrm{~mm}$ and width $74.5 \mathrm{~mm}$ is composed of $5 \times 5$-unit cells made of Rogers RO3210 as shown in Figure 4(a). The total thickness of $5.12 \mathrm{~mm}$ was obtained by superposing 4 identical dielectric layers of thickness $1.28 \mathrm{~mm}$. Each unit cell of size $L_{x} \times L_{y}$ $\left(L_{x}=14.9 \mathrm{~mm}, L_{y}=14.9 \mathrm{~mm}\right)$ contains two connected dielectric resonators with slots. We have considered the metasurface configuration with optimized design parameters. Therefore, the minor and major radii of the elliptic resonator of ellipticity $\tau=2.33$ are $a=3 \mathrm{~mm}(\approx \lambda / 5)$ and $b=7 \mathrm{~mm}(\approx \lambda /$ $2.14)$, respectively, whereas the minor and major radii of the elliptic slot of ellipticity $\tau_{\mathrm{s}}=2$ are $a_{\mathrm{s}}=2 \mathrm{~mm}(\approx \lambda / 7.5)$ 

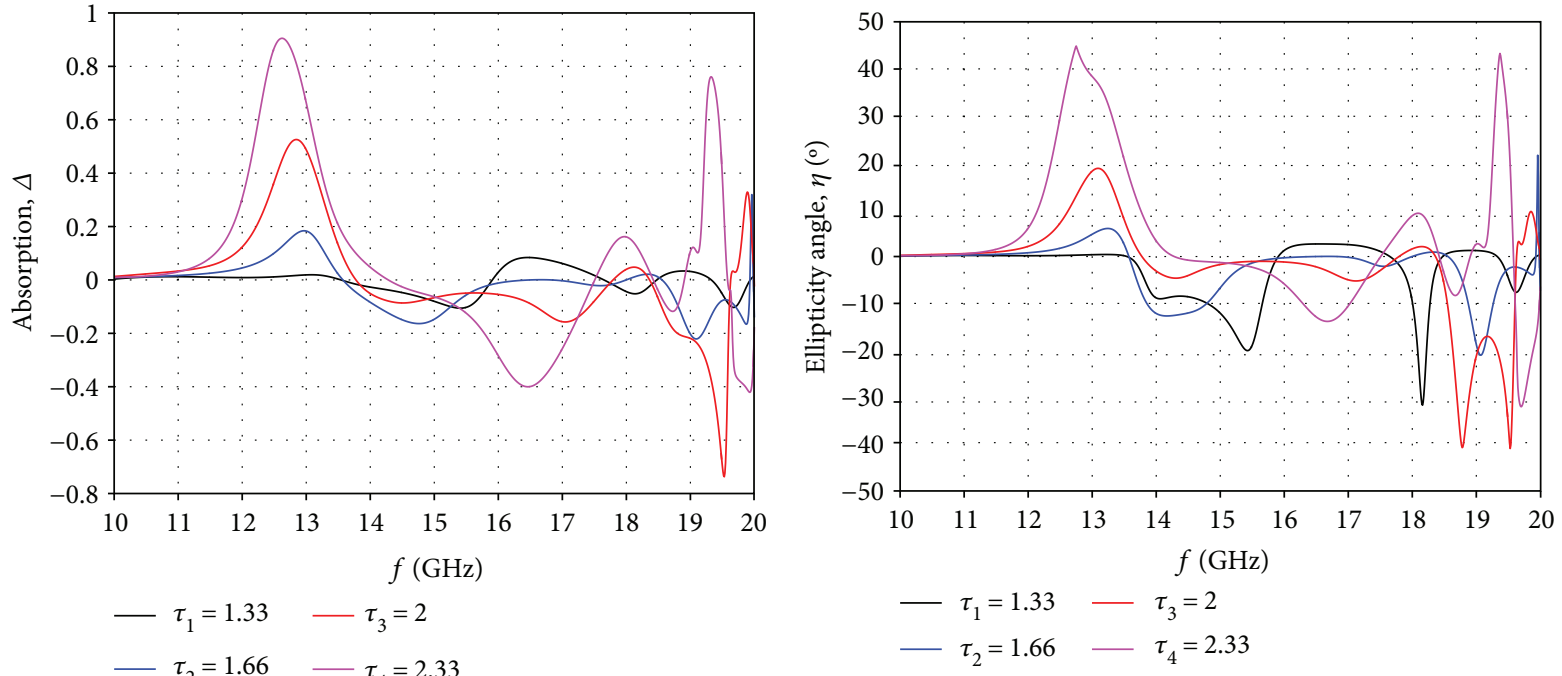

(a)

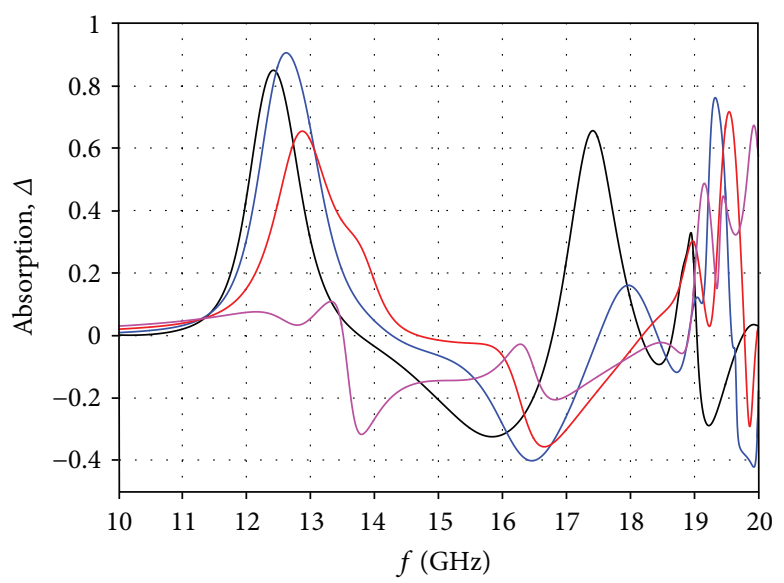

(b)

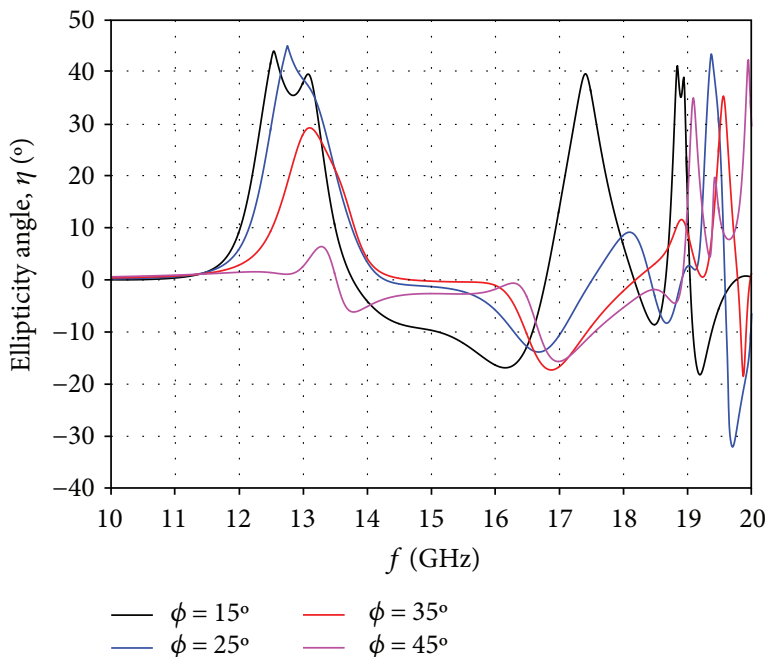

(c)

(d)

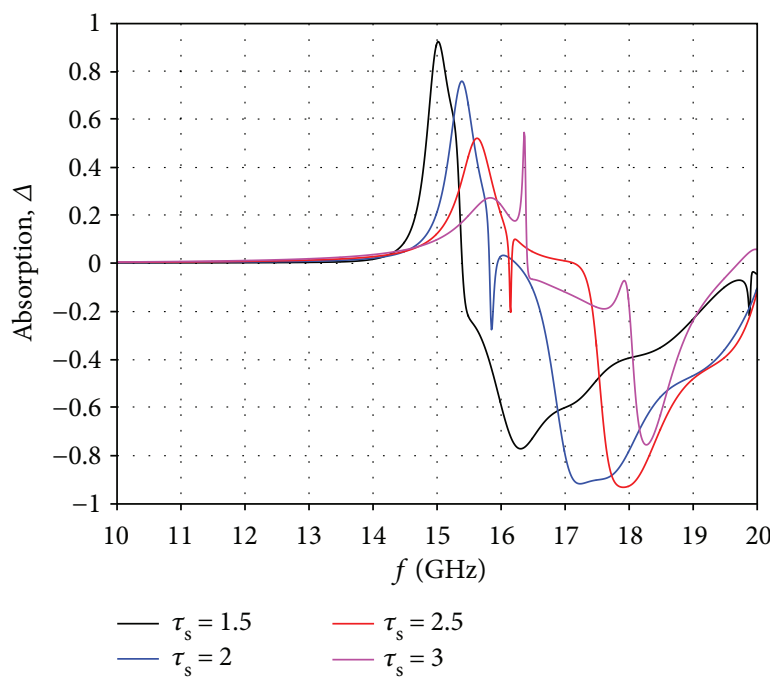

(e)

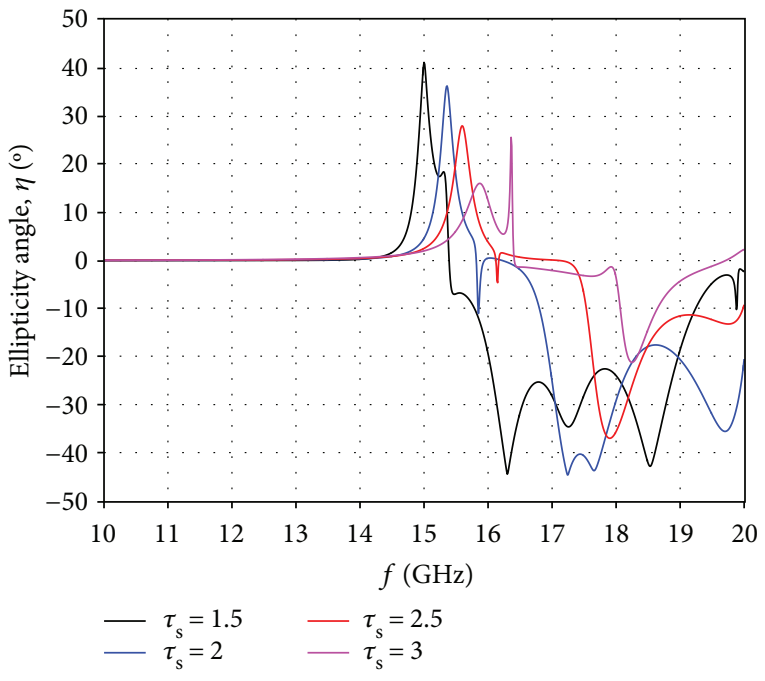

(f)

FIGURE 3: Variation of the absorption $\Delta$ and ellipticity angle $\eta$, over the frequency band $10-20 \mathrm{GHz}$, for different values of the resonator ellipticity ((a) and (b)) and orientation ((c) and (d)) and the slot ellipticity ((e) and (f)). 
TABle 1: Main data related to the effects of the resonator ellipticity (a), orientation (b), and slot ellipticity (c), on the metasurface circular dichroism.

(a)

\begin{tabular}{|c|c|c|c|c|c|c|}
\hline \multirow{3}{*}{ Frequency bandwidth for $|\Delta|>0.5$} & \multicolumn{6}{|c|}{ Resonator ellipticity, $\tau$} \\
\hline & \multirow{2}{*}{$\frac{1.33}{-}$} & \multirow{2}{*}{$\frac{1.66}{-}$} & \multicolumn{2}{|c|}{2} & \multicolumn{2}{|c|}{2.33} \\
\hline & & & $12.72-12.97$ & $19.41-19.6$ & $12.18-13.14$ & $19.22-19.48$ \\
\hline $\mathrm{Bw}(\%)$ & - & - & 1.9 & 0.9 & 7.5 & 1.3 \\
\hline Absorption for $|\Delta|>0.5$ & - & - & 0.52 & 0.73 & 0.9 & 0.76 \\
\hline Peak frequency, $f(\mathrm{GHz})$ & - & - & 12.85 & 19.53 & 12.62 & 19.32 \\
\hline Ellipticity angle, $\eta\left(^{\circ}\right)$ & - & - & 15.42 & -41 & 38.13 & 36.29 \\
\hline
\end{tabular}

(b)

\begin{tabular}{|c|c|c|c|c|c|c|c|}
\hline \multirow[b]{2}{*}{ Frequency bandwidth for $|\Delta|>0.5$} & \multicolumn{2}{|c|}{$\phi=15^{\circ}$} & \multicolumn{2}{|c|}{$\begin{array}{l}\text { Rotation angle, } \phi \\
\phi=25^{\circ}\end{array}$} & \multicolumn{2}{|c|}{$\phi=35^{\circ}$} & \multirow{2}{*}{$\frac{\phi=45^{\circ}}{19.83-20}$} \\
\hline & $12.05-12.82$ & $17.2-17.63$ & $12.18-13.14$ & $19.22-19.48$ & $12.59-13.21$ & $19.42-19.66$ & \\
\hline $\mathrm{Bw}(\%)$ & 6.1 & 2.4 & 7.5 & 1.3 & 4.8 & 1.2 & 0.8 \\
\hline Absorption for $|\Delta|>0.5$ & 0.84 & 0.65 & 0.9 & 0.76 & 0.65 & 0.71 & 0.67 \\
\hline Peak frequency, $f(\mathrm{GHz})$ & 12.42 & 17.41 & 12.62 & 19.32 & 12.87 & 19.54 & 19.93 \\
\hline Ellipticity angle, $\eta\left(^{\circ}\right)$ & 36.95 & 39.51 & 38.13 & 36.29 & 23.85 & 34.27 & 39.5 \\
\hline
\end{tabular}

(c)

\begin{tabular}{|c|c|c|c|c|c|c|c|c|}
\hline \multirow{3}{*}{$\begin{array}{l} \\
\text { Frequency bandwidth } \\
\text { for }|\Delta|>0.5\end{array}$} & \multicolumn{8}{|c|}{ Slot ellipticity, $\tau_{\text {s }}$} \\
\hline & \multicolumn{2}{|c|}{1.5} & \multicolumn{2}{|r|}{$\mathrm{T}$} & \multicolumn{2}{|c|}{2.5} & \multicolumn{2}{|c|}{3} \\
\hline & $14.83-15.3$ & $15.92-17.45$ & $15.21-15.58$ & $16.82-18.76$ & $15.56-15.69$ & $17.54-18.92$ & $16.35-16.37$ & $18.1-18.62$ \\
\hline $\mathrm{Bw}(\%)$ & 3.1 & 9.1 & 2.4 & 10.9 & 0.8 & 7.5 & 0.1 & 2.8 \\
\hline Absorption for $|\Delta|>0.5$ & 0.92 & 0.77 & 0.75 & 0.91 & 0.52 & 0.93 & 0.54 & 0.75 \\
\hline Peak frequency, $f(\mathrm{GHz})$ & 15.02 & 16.3 & 15.39 & 17.24 & 15.63 & 17.91 & 16.36 & 18.27 \\
\hline Ellipticity angle, $\eta\left(^{\circ}\right)$ & 39.5 & -44.44 & 33.6 & -44.6 & 26.87 & 36.96 & 25.67 & -21.1 \\
\hline
\end{tabular}

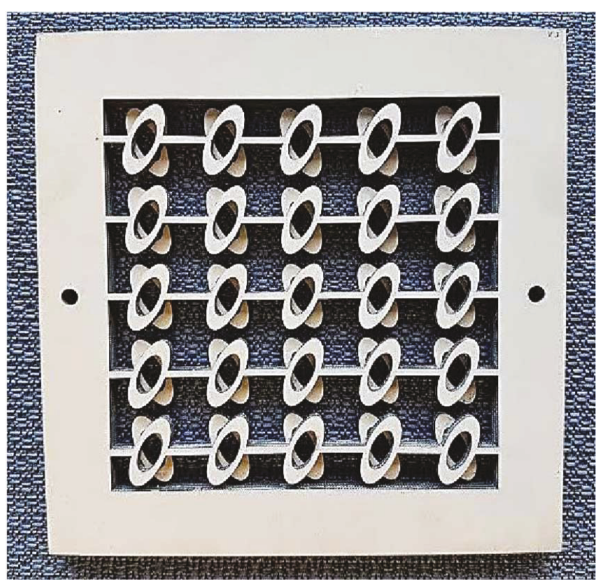

(a)

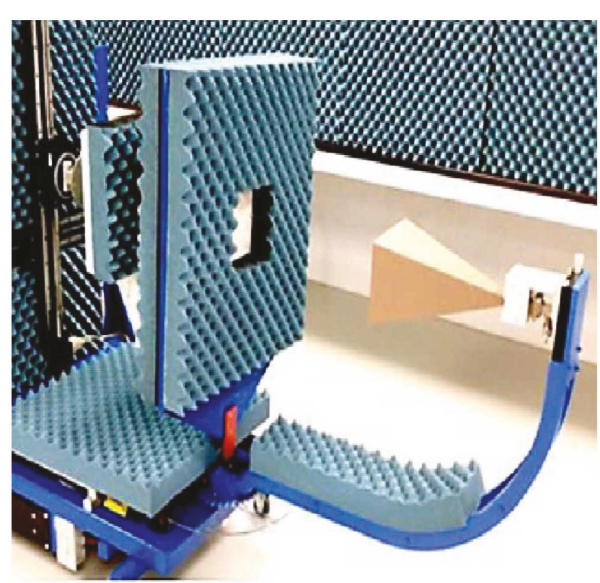

(b)

FIgURe 4: Photos of the realized prototype (a) and measurement setup (b).

and $b_{\mathrm{s}}=4 \mathrm{~mm}(\approx \lambda / 3.75)$, respectively. The dielectric strips ensuring the connection between resonators along the $x$ direction are of width $W=1.25 \mathrm{~mm}(\approx \lambda / 12)$. The rotation angles are $\phi=-25^{\circ}$ and $\phi=+25^{\circ}$ for all the resonators composing the upper and lower metasurface layers, respectively.
We have measured the transmission coefficients in terms of amplitude and phase by using the home-made measurement setup (developed by Dr. Christophe Caloz's research team at the University of Montreal in Canada) shown in Figure 4(b). This setup is composed with a rotating arm used 


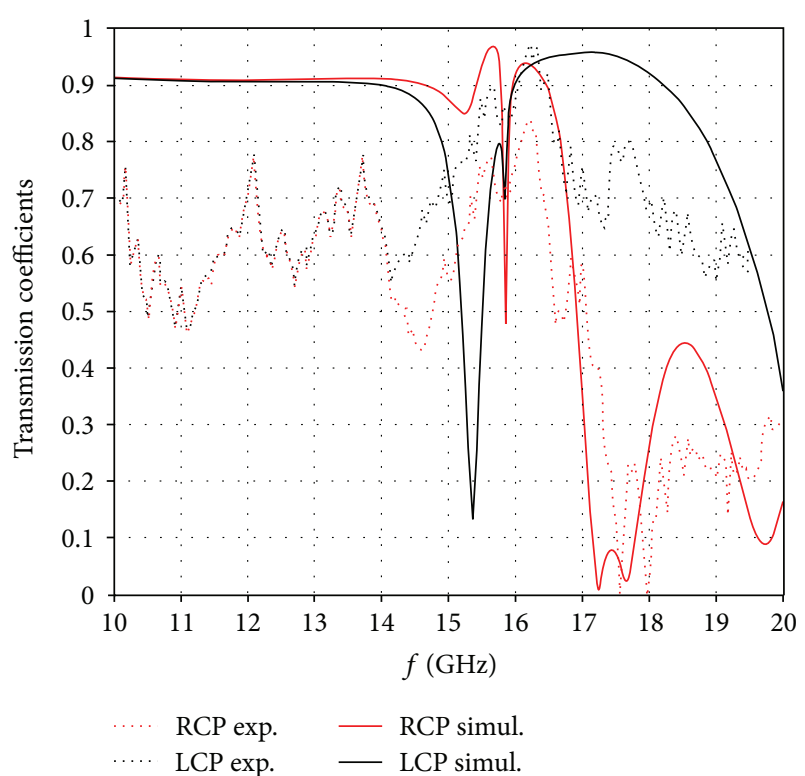

FIgURE 5: Variation of the simulated and measured RCP and LCP transmission coefficients over the frequency band $10-20 \mathrm{GHz}$.

to attach the exciting horn antenna, which can rotate from $-90^{\circ}$ to $+90^{\circ}$ around the center where the metasurface is placed. This arm is with sufficient length to ensure far-field measurements. On the other side of the setup, a moving scanning probe was placed to measure the transmitted power through the metasurface. The distance between the horn antenna and the device under test is chosen equal to $80 \mathrm{~mm}$, to perform measurement in the far-field region.

In Figure 5, we have superposed moduli of both simulated and measured RCP and LCP transmitted coefficients for the realized prototype over the frequency band 10$20 \mathrm{GHz}$. As it can be remarked, the agreement between simulation and measurement is acceptable over the majority part of the band $10-20 \mathrm{GHz}$, except the frequency range $14-15.8 \mathrm{GHz}$ where repetitive experiments fail to retrieve the predicted peak around $15.38 \mathrm{GHz}$. Analysis of the measured curves presented in Figure 5 shows that the RCP and LCP moduli are equal up to $14.1 \mathrm{GHz}$, whereas for higher frequencies, a visible difference between them is observed, which means that the circular dichroism of the proposed all-dielectric metasurface appears for frequencies higher than $14.1 \mathrm{GHz}$ with a tendency to transmit LCP waves more than RCP ones.

To better analyze the circular dichroism behavior, we have represented in Figure 6 both simulated and measured constitutive parameters, $\Delta$ and $\eta$ versus the frequency. Again, the predicted peak around $15.38 \mathrm{GHz}$ is not retrieved experimentally. However, we can notice that, for an absorption difference $\Delta>0.5$ between measured RCP and LCP waves with ellipticity close to $45^{\circ}$, the metasurface presents two operating bands $17.55-17.61 \mathrm{GHz} \quad(0.34 \%)$ and $17.91-$ $18.05 \mathrm{GHz}(0.77 \%)$ with maximum absorptions $\Delta=0.61$ and $\Delta=0.5$ and the maximum of ellipticities $\eta \approx-44.9^{\circ}$ and $\eta \approx-44.9^{\circ}$ at the peak frequencies 17.56 and $18 \mathrm{GHz}$, respectively. However, in the simulations (see Table 1),

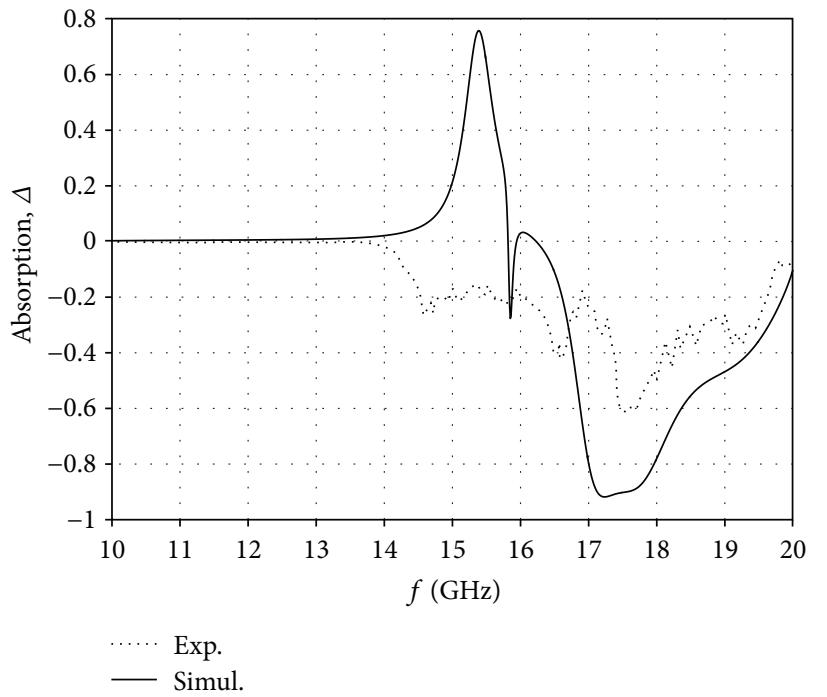

(a)

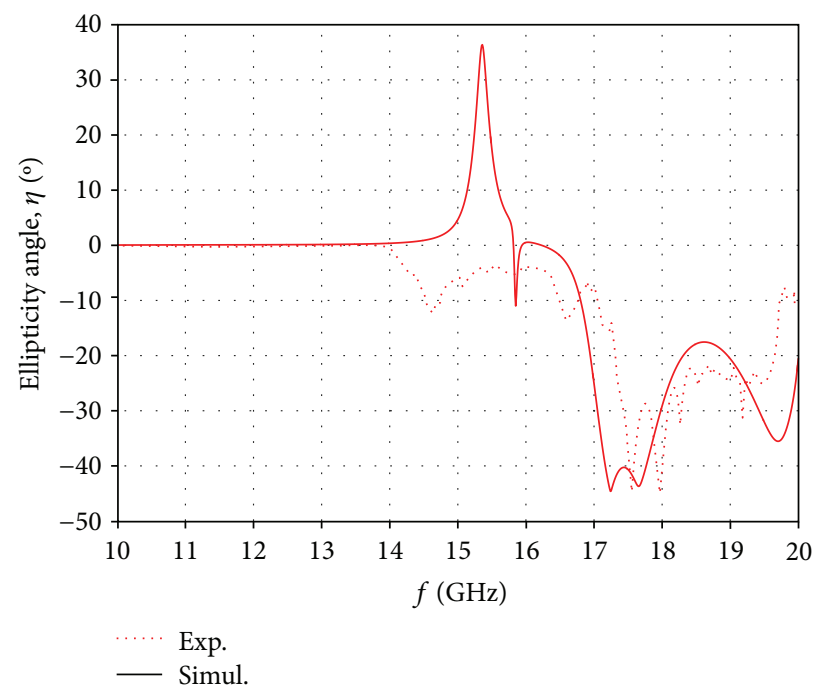

(b)

FIGURE 6: Variation of the simulated and measured absorption $\Delta$ (a) and ellipticity angle $\eta$ (b) over the frequency band 10-20 GHz.

we have obtained a unique wide band extending from 16.82 to $18.76 \mathrm{GHz}(10.9 \%)$ with a maximum of absorption $\Delta=0.91$ and ellipticity $\eta \approx-44.6^{\circ}$ at the resonant frequency $17.24 \mathrm{GHz}$. We estimate that the simulations are in acceptable agreement with the experiments. Finally, we can notice that the particular values (close to $45^{\circ}$ ) of the large ellipticities $\left(\eta \approx-44.9^{\circ}\right.$ at $17.56 \mathrm{GHz}$ and $\eta \approx-44.9^{\circ}$ at $\left.18 \mathrm{GHz}\right)$ mean that the transmitted waves through the all-dielectric metasurface are circularly polarized, which may offer to the metadevice the possibility to be used as a circular polarization filter and circular polarizer at microwave frequencies.

\section{Conclusion}

An all-dielectric chiral metasurface with circular dichroism behavior was designed to operate at microwave frequencies. The structure based on elliptic dielectric resonators was first 
optimized numerically by studying the effects of the main design parameters on its transmission within the band 10$20 \mathrm{GHz}$. The optimized design was fabricated and tested experimentally, and the measured results were compared with simulations. It is found that the fabricated prototype is characterized by a large circular dichroism over two bands with ellipticities close to $45^{\circ}$.

\section{Conflicts of Interest}

The authors declare that there is no conflict of interests regarding the publication of this article.

\section{Acknowledgments}

This project was funded by the Deanship of Scientific Research (DSR), King Abdulaziz University, Jeddah, under Grant no. RG-2-135-38. The authors, therefore, acknowledge with thanks DSR for technical and financial support.

\section{References}

[1] F. Capolino, Ed., Theory and Phenomena of Metamaterials, CRC, Boca Raton, FL, USA, 2009.

[2] J. B. Pendry, "Negative refraction makes a perfect lens," Physical Review Letters, vol. 85, no. 18, pp. 3966-3969, 2000.

[3] J. B. Pendry, D. Schurig, and D. R. Smith, "Controlling electromagnetic fields," Science, vol. 312, no. 5781, pp. 1780-1782, 2006.

[4] A. Silva, F. Monticone, G. Castaldi, V. Galdi, A. Alú, and N. Engheta, "Performing mathematical operations with metamaterials," Science, vol. 343, no. 6167, pp. 160-163, 2014.

[5] C. L. Holloway, M. A. Mohamed, E. F. Kuester, and A. Dienstfrey, "Reflection and transmission properties of a metafilm: with an application to a controllable surface composed of resonant particles," IEEE Transactions on Electromagnetic Compatibility, vol. 47, no. 4, pp. 853-865, 2005.

[6] C. L. Holloway, E. F. Kuester, J. A. Gordon, J. O’Hara, J. Booth, and D. R. Smith, "An overview of the theory and applications of metasurfaces: the two-dimensional equivalents of metamaterials," IEEE Antennas and Propagation Magazine, vol. 54, no. 2, pp. 10-35, 2012.

[7] H. T. Chen, A. J. Taylor, and N. Yu, "A review of metasurfaces: physics and applications," Reports on Progress in Physics, vol. 79, no. 7, article 076401, 2016.

[8] K. Achouri, M. A. Salem, and C. Caloz, "General metasurface synthesis based on susceptibility tensors," IEEE Transactions on Antennas and Propagation, vol. 63, no. 7, pp. 2977-2991, 2015.

[9] A. Arbabi, Y. Horie, M. Bagheri, and A. Faraon, "Dielectric metasurfaces for complete control of phase and polarization with subwavelength spatial resolution and high transmission," Nature Nanotechnology, vol. 10, no. 11, pp. 937-943, 2015.

[10] K. Achouri, G. Lavigne, M. A. Salem, and C. Caloz, "Metasurface spatial processor for electromagnetic remote control," IEEE Transactions on Antennas and Propagation, vol. 64, no. 5, pp. 1759-1767, 2016.

[11] K. Achouri, A. Yahyaoui, S. Gupta, H. Rmili, and C. Caloz, "Dielectric resonator metasurface for dispersion engineering," IEEE Transactions on Antennas and Propagation, vol. 65, no. 2, pp. 673-680, 2017.
[12] I. Staude, A. E. Miroshnichenko, M. Decker et al., "Tailoring directional scattering through magnetic and electric resonances in subwavelength silicon nanodisks," ACS Nano, vol. 7, no. 9, pp. 7824-7832, 2013.

[13] Y. Dai, W. Ren, H. Cai, H. Ding, N. Pan, and X. Wang, "Realizing full visible spectrum metamaterial half-wave plates with patterned metal nanoarray/insulator/metal film structure," Optics Express, vol. 22, no. 7, pp. 7465-7472, 2014.

[14] J. Cheng, D. Ansari-Oghol-Beig, and H. Mosallaei, "Wave manipulation with designer dielectric metasurfaces," Optics Letters, vol. 39, no. 21, pp. 6285-6288, 2014.

[15] A. Yahyaoui, H. Rmili, M. Sheikh, A. Dobaie, L. Laadhar, and T. Aguili, "Design of all-dielectric half-wave and quarter-wave plates microwave metasurfaces based on elliptic dielectric resonators," Applied Computational Electromagnetics Society Journal, vol. 32, no. 3, pp. 229-236, 2017.

[16] A. Yahyaoui, H. Rmili, K. Achouri, M. Sheikh, A. Dobaie, and T. Aguili, "Transmission control of electromagnetic waves by using quarter-wave plate and half-wave plate all-dielectric metasurfaces based on elliptic dielectric resonators," International Journal on Antennas and Propagation, vol. 2017, Article ID 8215291, 8 pages, 2017.

[17] Z. Wang, F. Cheng, T. Winsor, and Y. Liu, "Optical chiral metamaterials: a review of the fundamentals, fabrication methods and applications," Nanotechnology, vol. 27, no. 41, article 412001, 2016.

[18] G. Kenanakis, R. Zhao, A. Stavrinidis et al., "Flexible chiral metamaterials in the terahertz regime: a comparative study of various designs," Optical Materials Express, vol. 2, no. 12, pp. 1702-1712, 2012.

[19] J. Zhou, D. R. Chowdhury, R. Zhao et al., "Terahertz chiral metamaterials with giant and dynamically tunable optical activity," Physical Review B, vol. 86, no. 3, article 035448, 2012.

[20] F. Fang and Y. Cheng, "Dual-band terahertz chiral metamaterial with giant optical activity and negative refractive index based on cross-wire strucure," Progress in Electromagnetics Research M, vol. 31, pp. 59-69, 2013.

[21] Y. Cheng, Y. Nie, L. Wu, and R. Z. Gong, "Giant circular dichroism and negative refractive index of chiral metamaterial based on split-ring-resonators," Progress In Electromagnetics Research, vol. 138, pp. 421-432, 2013.

[22] I. de Leon, M. J. Horton, S. A. Schulz, J. Upham, P. Banzer, and R. W. Boyd, "Strong, spectrally-tunable chirality in diffractive metasurfaces," Scientific Reports, vol. 5, no. 1, article 13034, p. 9, 2015.

[23] X. Ma, M. Pu, X. Li et al., "A planar chiral meta-surface for optical vortex generation and focusing," Scientific Reports, vol. 5, no. 1, article 10365, p. 7, 2015.

[24] J. Hu, X. Zhao, Y. Lin et al., "All-dielectric metasurface circular dichroism waveplate," Scientific Reports, vol. 7, article 41893, p. 9, 2017.

[25] J. D. Jackson, Classical Electrodynamics, Wiley, New York, NY, USA, 3rd edition, 1999. 


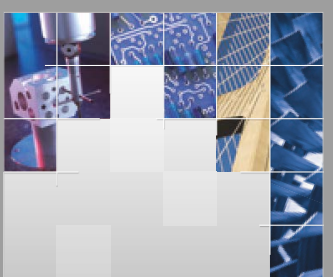

\section{Enfincering}
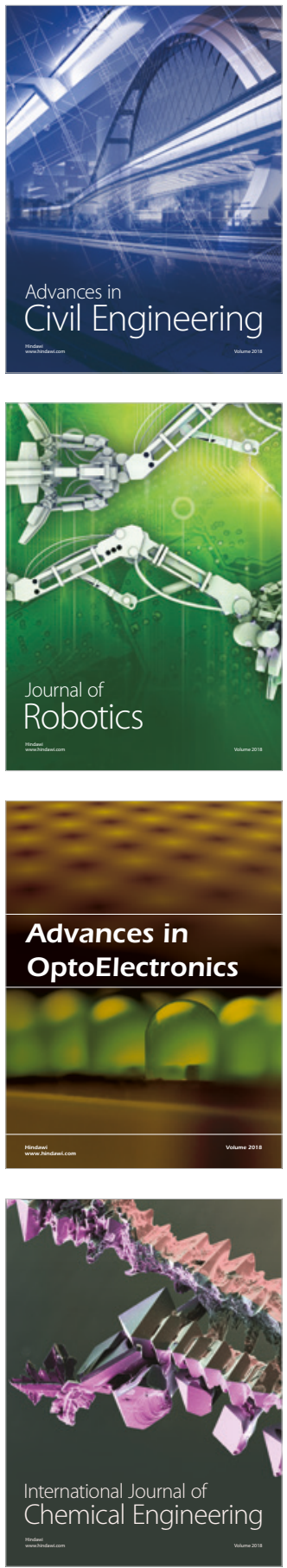

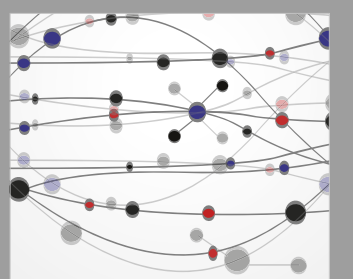

\section{Rotating \\ Machinery}

The Scientific World Journal

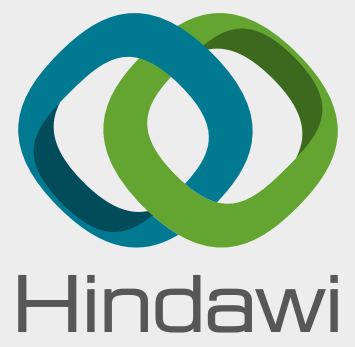

Submit your manuscripts at

www.hindawi.com
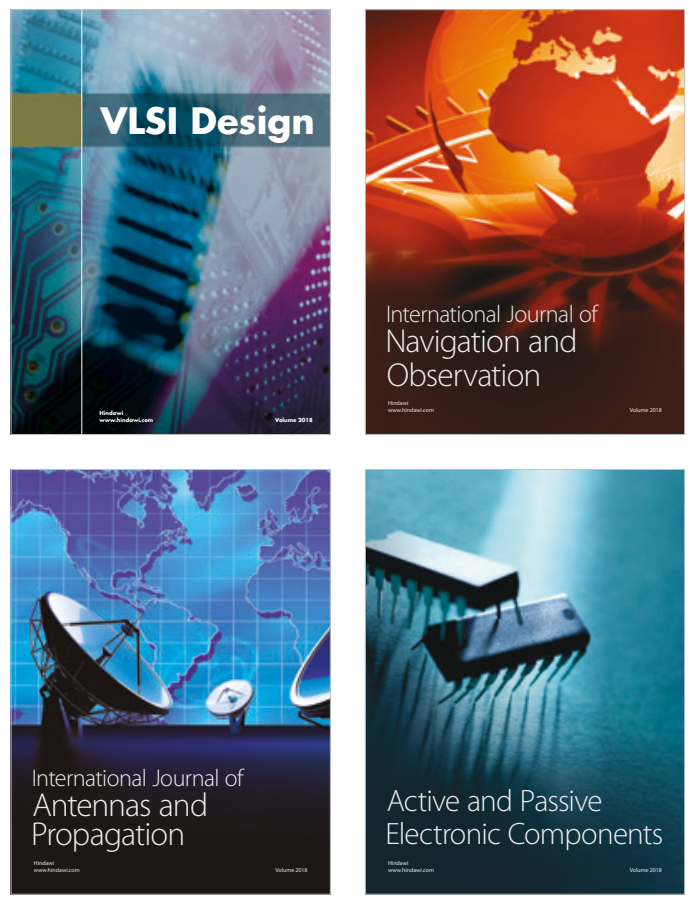
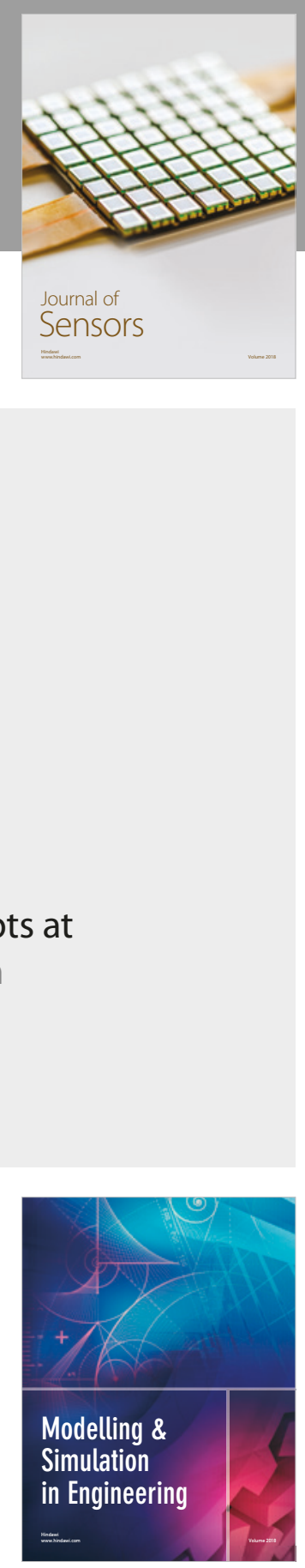

\section{Advances \\ Multimedia}
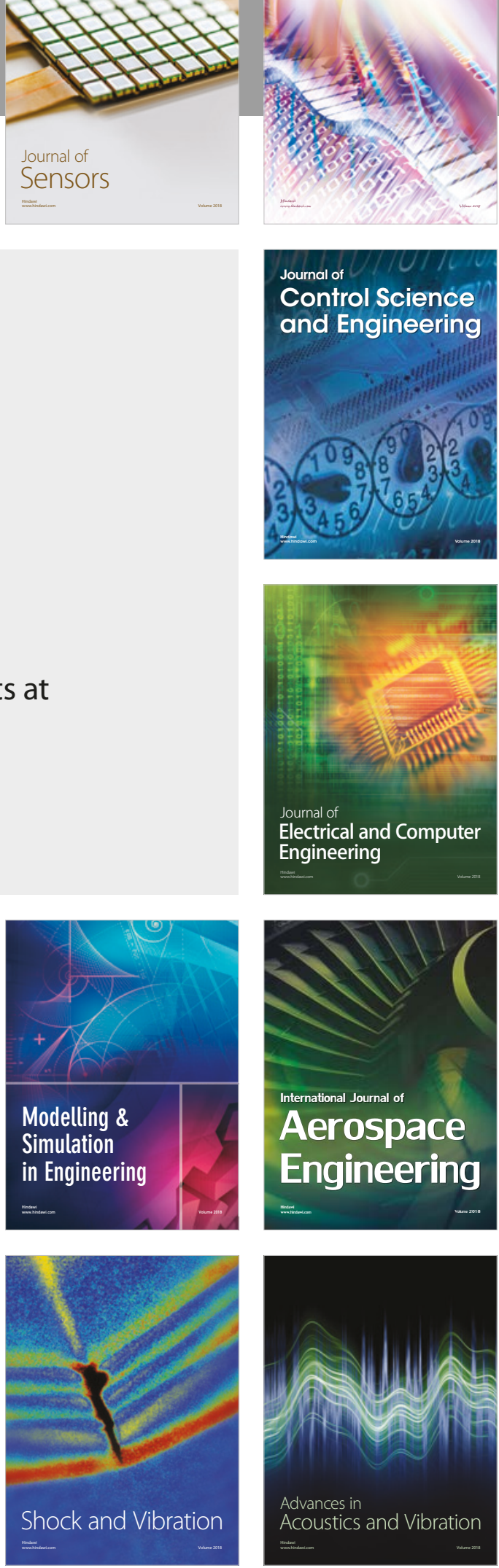